negative history will significantly decrease the number of women who receive the vaccine. The proportion of women who would be vaccinated, based on a negative history without serological confirmation, would vary widely between different populations as the number of adults who could not recall a history of chickenpox ranged from $19 \%$ in $\mathrm{Ohio}^{12}$ to $49 \%$ in Australia. ${ }^{10}$ However, even among subjects with no, or uncertain, prior exposure to varicella, only $10-36 \%$ were non-immune. ${ }^{11} 12$

The availability of an active varicella vaccine opens new options for preventing chickenpox in pregnancy. Moreover, doctors may possibly face medicolegal consequences if they fail to offer the vaccine during preconception consultation. The attack rate of varicella is extremely high with household contact, and a substantial number of women with a negative history are susceptible. Furthermore, women of childbearing age may be more exposed to chickenpox from non-immunised children in their household. A routine screening and vaccination programme, similar to the one currently implemented for rubella, must be considered. A history of chickenpox should be obtained from all women at preconception counselling. Serological testing or vaccination should be offered. However, women should be informed that if they have a history of varicella there is at least a $\mathbf{9 7 \%}$ chance that they are immune and that even among those who can not recall varicella infection about $80 \%$ are immune. The cost effectiveness of vaccinating women of childbearing age should be determined for each population according to its rates of seronegativity. Future vaccination policies would need to be revised in areas where all children are now routinely immunised as recommended.

DANIEL S SEIDMAN Research fellow

Department of Obstetrics and Gynecology,

Sheba Medical Center,

Tel-Hashomer,

Israel

DAVID K STEVENSON

Professor of neonatology

ANN M ARVIN

Department of Pediatrics, Professor of infectious diseases

Stanford University Medical School,

Stanford, CA, 94305-5119,

USA
1 Gershon AA, Raker R, Steinberg S, Topf-Olstein B, Drusin LM. Antibody to varicella-zoster in parturient women and their offspring in the first year of life. Pediatrics 1976;58:692-6.

2 Paryani S, Arvin A. Intrauterine infection with varicella-zoster virus after maternal varicella. $N$ Engl F Med 1986;314:1542-6.

$3 \mathrm{Kaz}$ VL, Kuller JA, McMahon MJ, Warren MA, Wells SR. Varicella during pregnancy. Maternal and fetal effects. West $\mathcal{F} M$ Med $1995 ; 163: 446-50$.

4 Pastuszak AL, Levy M, Schick B, Zuber C, Feldkaamp M, Gladstone J, et al. Outcome after

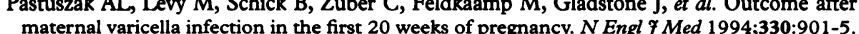
Miller E, Cradock-Watson JE, Pollock TM. Consequences of confirmed maternal rubella at successive stages of pregnancy. Lancet 1982;ii:781-4.

6 Enders G, Miller E, Cradock-Watson J, Bolley J, Ridehalgh M. Consequences of varicella and herpes zoster in pregnancy: prospective study of 1739 cases. Lancet 1994;343:1547-50.

Perinatal viral and parasitic infections. ACOG technical bulletin number 177, February 1993 Int $\mathcal{F}$ Gynecol Obstet 1993;42:300-7.
8 Lecuru F, Taurelle R, Bernard JP, Parrat S, Lafay-pillet MC, Rozenberg F, et al. Varicella zoster infection during pregnancy; the limits of prenatal diagnosis. Eur $f$ Obstet Gynecol 1994;56:67-8.

9 Gershon AA, Steinberg SP, the National Institute of Allergy and Infectious Disease Varicella Vaccine Collaborative Study Group. Live attenuated varicella vaccine: protection in healthy adults compared with leukemic children. I Infect Dis 1990;158:661-6.

10 Rouse DJ, Gardner M, Allen SJ, Goldenberg RL. Management of the presumed susceptible varicella (chickenpox)-exposed gravida: a cost-effective/cost-benefit analysis. Obstet Gynecol varicella (chick $1996 ; 87: 932-6$.

11 Ferson MJ, Bell SM, Robertson PW. Determination and importance of varicella immune status of nursing staff in a children's hospital. $\mathcal{F}$ Hosp Infect 1990;15:347-51.

12 Struewing JP, Hymas KC, Tueller JE, Gray GC. The risk of measles, mumps, and varicella among young adults: a serosurvey of US Navy and Marine Corps recruits. Am $\mathcal{Y}$ Public Health 1993;83:1717-20

\title{
Growing pains of meta-analysis
}

\section{Advances in methodology will not remove the need for well designed trials}

Meta-analysis is a statistical technique for pooling clinical trials of similar design. It achieves higher statistical power for detecting differences between treatments and obtaining more precise estimates of outcome. Meta-analysis quickly chalked up some notable successes after its introduction to medicine. Meta-analysis of several small studies suggested thrombolytic treatment would reduce mortality from acute myocardial infarction by $20 \%,{ }^{1}$ a prediction confirmed by subsequent large randomised trials. ${ }^{2}$ The value of the meta-analysis in providing a quantitative overview of data from many studies soon became apparent. Optimists suggested that cumulative meta-analysis of small trials would identify effective treatments sooner, ${ }^{3}$ and there were even hints that meta-analysis might make large randomised trials unnecessary.

But recent discrepancies between the predictions of metaanalysis and findings of large randomised trials have been disappointing and have led to a critical reappraisal of this technique. Meta-analysis of eight small randomised studies suggested intravenous magnesium would reduce the risk of acute myocardial infarction by $55 \%(P<0.001),{ }^{45}$ but a randomised trial of 58050 patients found no evidence of efficacy. ${ }^{6}$

This discrepancy between the predictions of meta-analysis and the results of a mega-trial might be due to publication bias, to differences in patient populations, to the dose and timing of intravenous magnesium, or to greater use of other effective treatments such as fibrinolysis. Similar predictions that nitrates would reduce mortality from acute myocardial infarction ${ }^{7}$ were not borne out by trials of 18895 and 58050 patients. $^{68}$ Meta-analysis of small randomised trials in perinatal medicine had only moderate agreement ( $\kappa 0.46-0.53)$ with large trials of more than 1000 patients. $^{9}$ These recent failures have led to considerable discussion and criticism of meta-analysis.

Meta-analyses can suffer from incomplete reporting of data, variation in the quality of studies, and bias in selecting which studies to include. Meta-analyses that use only published data can also be biased by the preferential publication of positive trials. The actual synthesis of information from independent studies is also limited by differences in study design, intervention, and patient population. With all these potential difficulties, a meta-analysis should make a strong case for why certain trials should be pooled, and should explore the quantitative effect of known differences in trial design or patient populations on the summary outcome. Since standard meta-analytic methods have not often incorporated study level or patient level covariates into their quantitative analysis, newer statistical methods that can assess the independent and joint effects of covariates on the overall outcome are needed. On $\mathrm{p} 735$ Sharp et al discuss some of the problems in meta-analyses. ${ }^{10}$ These newer methods would be particularly important for synthesising non-experimental data, and would allow meta-analysis to better fulfill its promise as a "study of studies."

Advances in meta-analytic methods will not remove the need for well designed trials, and may in fact highlight the importance of good trial design for avoiding systemically 
biased outcomes. Furthermore, pooling of well reported primary data from trials can show how much outcomes vary, if at all, with different treatments and different characteristics for individual patients.

Meta-analysis is a valuable technique. Like many innovations in medicine, it has gone through a cycle of optimism, inflated promises, and disappointment; as it reaches maturity, a more balanced view of its strengths and limitations is emerging.

IDA SIM

Postdoctoral fellow

MARK A HLATKY

Professor

Department of Health Research and Policy,

Department of Medicine,

Stanford University School of Medicine,

Stanford, CA 94305-5092,

USA

Supported in part by Grant HS 08362 from the Agency for Health Care Policy and Research, Rockville, MD.
1 Stampfer MJ, Goldhaber SZ, Yusuf S, Peto R, Hennekens CH. Effect of intravenous streptokinase on acute myocardial infarction. Pooled results from randomized trials. $N$ Engl $f$ Med 1982;307:1180-2.

2 Rovelli F, DeVita C, Feruglio GA, Lotto A, Selvini A, Tognoni G. Effectiveness of intravenous thrombolytic treatment in acute myocardial infarction. Lancet 1986;i:397-402.

3 Antman EM, Lau J, Kupelnick B, Mosteller F, Chalmers TC. A comparison of results of metaanalyses of randomized control trials and recommendations of clinical experts. Treatment for myocardial infarction. $¥ A M A$ 1992;268:240-8.

4 Yusuf S, Teo K, Woods K. Intravenous magnesium in acute myocardial infarction. An effective, safe, simple, and inexpensive intervention. Circulation 1993;87:2043-6.

5 Horner SM. Efficacy of intravenous magnesium in acute myocardial infarction in reducing arrhythmias and mortality. Meta-analysis of magnesium in acute myocardial infarction. Circulation 1992;86:774-8.

6 ISIS-4 (Fourth International Study of Infarct Survival) Collaborative Group. ISIS-4: A randomised factorial trial assessing early oral captopril, oral mononitrate, and intravenous magnesium sulphate in 58050 patients with suspected acute myocardial infarction. Lancet magnesium sulph

7 Yusuf S, MacMahon S, Collins R, Peto R. Effect of intravenous nitrates on mortality in acute myocardial infarction: an overview of the randomised trials. Lancet 1988;i:1088-92.

8 Gruppo Italiano per lo Studio della Sopravvivenza nell'Infarto Miocardico. GISSI-3: Effects of lisinopril and transdermal glyceryl trinitrate singly and together on 6-week mortality and ventricular function after acute myocardial infarction. Lancet 1994;343:1115-22.

9 Villar J, Carroli G, Belizan JM. Predictive ability of meta-analyses of randomised controlled trials. Lancet 1995;345:772-6.

10 Sharp SJ, Thompson SG, Altman DG. The relation between treatment benefit and underlying risk in meta-analyses. BMF 1996;313:735-8.

\title{
Vascularised pancreas transplantation
}

\author{
The ultimate treatment for insulin dependent diabetes
}

In vascularised pancreas transplantation the organ is transplanted as a whole (as opposed to islet cell transplantation, which is still experimental). The procedure re-establishes endogenous secretion of insulin that is responsive to normal feedback controls and is currently the only known treatment for diabetes that reliably achieves a euglycaemic state with complete normalisation of glycated haemoglobin concentrations. ${ }^{1}$ The costs that must be paid for normal glucose homeostasis are the operative risks of the procedure and the need for chronic immunosuppressive treatment.

Between 1966 and 1995 over 7500 pancreas transplantations were performed worldwide and reported to the International Pancreas Transplant Registry. ${ }^{2}$ Most (87\%) of these organs were transplanted in conjunction with kidneys as combined pancreatic and renal transplants into patients with impending or actual renal failure. The other operations included transplantation of the pancreas after the kidney (7.4\%), pancreas transplantation done alone $(5 \%)$, and pancreas transplantation in conjunction with a single organ other than the kidney or with multiple organs (less than $1 \%$ ). The total number of pancreas transplantations done each year has steadily increased, reaching over 1100 in $1995 .^{2}$ The overall one year actuarial survival of patients and of functional pancreas grafts (complete insulin independence) are $91 \%$ and $75 \%$ respectively.

Most doctors will consider a combined pancreatic and renal transplant for patients with insulin dependent diabetes mellitus and advanced nephropathy (creatinine clearance below $30 \mathrm{ml} / \mathrm{min}$ or failure of a prior kidney transplant). ${ }^{13}$ Such patients who also have minimal or limited secondary complications of diabetes are considered to be the best candidates. Most are between 20 and 40 years old. The selection criteria for a solitary pancreas transplantation are based on substantial impairment of quality of life associated with the presence of early diabetic complications or exogenous insulin failure with hyperlabile diabetes. ${ }^{4}$ The main determinants for selecting recipients are the presence of diabetic complications, the degree of nephropathy, and the cardiovascular risk. ${ }^{3}$

Controversy persists, however, about the use of pancreas transplantation. There is no dispute that kidney transplantation is the treatment of choice for many patients with advanced diabetic nephropathy. ${ }^{5}$ The additional transplantation of the pancreas remains debatable because of concerns that it may increase the morbidity and mortality associated with kidney transplantation alone. ${ }^{6}$ Indeed, owing to the careful selection of donors and recipients, the registry data show that survival of kidney grafts is better in patients who have simultaneously received a pancreas transplant than in diabetic patients who received a kidney transplant alone. ${ }^{7}$ Current data show that the projected half life of a kidney of a combined pancreatic and renal transplant is $\mathbf{1 1 . 3}$ years, compared with a half life of 7.9 years for a cadaveric donor kidney transplanted alone. In spite of the increased risk of morbidity, a combined pancreatic and renal transplant in an appropriately selected diabetic patient does not seem to jeopardise either the patient or the kidney and may result in excellent survival of both, with greater potential for complete rehabilitation. ${ }^{18}$

Pancreas transplantation also has the potential to relieve some of the secondary complications of diabetes. ${ }^{14}$ 8-10 Preliminary evidence suggests that successful pancreas transplantation may induce regression of early (but not advanced) microscopic lesions of diabetic nephropathy and stabilise renal function. It will also prevent the recurrence of diabetic nephropathy in a patient's kidney graft. The course of diabetic retinopathy seems to be less favourably influenced by a functioning pancreas graft. However, data from longer follow up (more than four years) of recipients suggest that retinopathy may also be stabilised, and the same seems to be true for both peripheral and autonomic neuropathies. ${ }^{8-10}$ Improved nerve conduction velocity, gastric function, cardiac function, and microcirculatory blood flow have also been reported. ${ }^{9}$ Furthermore, a functioning pancreas graft may help to reduce the hyperlipidaemic effects of immunosuppression and actually improve lipid metabolism over time. However, long term studies are needed to document fully the effects of pancreas transplantation on diabetes.

In patients with hyperlabile diabetes, extreme difficulty with metabolic control, or unawareness of hypoglycaemia a pancreas transplant can immediately transform the patient's quality of life simply by abolishing dependence on insulin and improving counterregulation. ${ }^{11}$ Changes in carbohydrate and lipid metabolism should also translate into a decreased risk of atherosclerotic vascular disease. 